University of Wollongong

Research Online

Faculty of Engineering and Information

Faculty of Engineering and Information

Sciences - Papers: Part A

Sciences

$1-1-2015$

\title{
Time-dependent mechanical properties of tough ionic-covalent hybrid hydrogels
}

\author{
Hai Xin \\ University of Wollongong, hxin@uow.edu.au \\ Hugh Ralph Brown \\ University of Wollongong, hbrown@uow.edu.au \\ Sina Naficy \\ University of Wollongong, snaficy@uow.edu.au \\ Geoffrey M. Spinks \\ University of Wollongong, gspinks@uow.edu.au
}

Follow this and additional works at: https://ro.uow.edu.au/eispapers

Part of the Engineering Commons, and the Science and Technology Studies Commons 


\title{
Time-dependent mechanical properties of tough ionic-covalent hybrid hydrogels
}

\begin{abstract}
Hybrid gels featuring interpenetrating covalent and ionic crosslinked networks have recently been shown to exhibit both high toughness and recoverability of strain-induced network damage. The high toughness results from the energy dissipated as entropically strained network strands are released by the dissociation of ionic crosslinks. As in the so-called double network hydrogels, the toughening process is inherently linked to network damage. This damage, however, can be recovered to a large degree in hybrid gels due to the reformation of ionic associations when the gel is unloaded. The stability of the ionic network under load is here investigated and it is shown that these networks show large stress relaxation at constant strain, time dependent stress-strain behaviour and rate-dependent toughness. A double exponential model is invoked to mathematically describe the stress relaxation of the hybrid gels indicating at least two relaxation mechanisms. The rate-dependent toughness and the relaxation behaviour of the hybrid gels are attributed to the labile unzipping of the ionic crosslinks which is assumed to be load and time dependent.
\end{abstract}

\section{Keywords}

dependent, mechanical, properties, time, tough, hydrogels, ionic, covalent, hybrid

Disciplines

Engineering | Science and Technology Studies

\section{Publication Details}

Xin, H., Brown, H. R., Naficy, S. \& Spinks, G. M. (2015). Time-dependent mechanical properties of tough ionic-covalent hybrid hydrogels. Polymer, 65 253-261. 


\section{Time-Dependent Mechanical Properties of Tough}

\section{Ionic-Covalent Hybrid Hydrogels}

Hai Xin ${ }^{a}$, Hugh R. Brown ${ }^{a, b}$, Sina Naficy ${ }^{a, b}$ and Geoffrey M. Spinks ${ }^{* a, b}$

${ }^{a}$ ARC Centre of Excellence for Electromaterials Science and Intelligent Polymer Research Institute, University of Wollongong, Innovation Campus, Squires Way, North Wollongong, NSW, 2522, Australia.

${ }^{b}$ School of Mechanical, Materials and Mechatronic Engineering, University of Wollongong, Wollongong, NSW, 2522, Australia.

*to whom all the correspondence should be addressed

Tel+61 24220 3010; gspinks@uow.edu.au 


\section{Abstract}

Hybrid gels featuring interpenetrating covalent and ionic crosslinked networks have recently been shown to exhibit both high toughness and recoverability of strain-induced network damage. The high toughness results from the energy dissipated as entropically strained network strands are released by the dissociation of ionic crosslinks. As in the socalled double network hydrogels, the toughening process is inherently linked to network damage. This damage, however, can be recovered to a large degree in hybrid gels due to the reformation of ionic associations when the gel is unloaded. The stability of the ionic network under load is here investigated and it is shown that these networks show large stress relaxation at constant strain, time dependent stress-strain behaviour and rate-dependent toughness. A double exponential model is invoked to mathematically describe the stress relaxation of the hybrid gels indicating at least two relaxation mechanisms. The ratedependent toughness and the relaxation behaviour of the hybrid gels are attributed to the labile unzipping of the ionic crosslinks which is assumed to be load and time dependent.

Keyword: hybrid hydrogel, toughness, rate-dependence, stress relaxation.

\section{Introduction}

The super water-absorbing capabilities and external-stimuli responsiveness of hydrogels are of interest for a wide range of biological and bio-mechanical applications, such as sensors, actuators, and replacement materials for human tissues [1-3]. Unfortunately, many proposed applications are not realized due to the poor mechanical properties of conventional covalently-crosslinked hydrogels. Enhancing the mechanical performance of hydrogels has thereby become a central challenge and several breakthroughs have been achieved including double network hydrogel (DN gel) [4], clay-polymer nano-composite hydrogel (NC gel) [5], 
and slide-ring hydrogel (SR gel) [6]. These novel hydrogels exhibit remarkably-improved mechanical strength and toughness compared with the conventional hydrogels [7].

Recently, in het Panhuis et al. [8] and Suo et al. [9] have independently reported an interpenetrating polymer network hydrogel based on a covalently crosslinked polyacrylamide (PAAm) and an ionically crosslinked biopolymer (gellan gum in the former study and alginate in the latter). Since the hydrogels combined two interpenetrating networks with different crosslinking types, they were referred to as "ionic-covalent entanglement" hydrogel (ICE) [8] or a "hybrid gel" [9]. Both hydrogel systems exhibit high toughness and recoverability in mechanical properties, which is a major advantage over other tough hydrogels that are permanently damaged during loading and unsuitable for applications where repeated loading and unloading is required.

When the network topologies of PAAm-alginate hybrid gel were optimized, the resulting hydrogel was found to be highly stretchable (extended to $>20$ times its original length), very tough (fracture energy reaches $9000 \mathrm{~J} / \mathrm{m}^{2}$ ), and recoverable in mechanical properties. Mechanical hysteresis is a feature of these materials wherein the loading and unloading tensile curves are different. Importantly, it has been shown that a rest period after unloading allows the hybrid gels to recover and reloading follows closely the previous loading path. In contrast, the reloading curves in DN gels follow the previous unloading curve indicating the permanent nature of the damage occurring in these covalently crosslinked gels. The high toughness and mechanical recoverability of the hybrid gel system originates from the ionic crosslinks, where the guluronic units (also known as G group) on the alginate chains are associated by $\mathrm{Ca}^{2+}$ ions to form a zip-like junction described by a socalled "egg-box" model [10-13]. When a hybrid gel is subject to external force the crosslinks are "unzipped", dissipating dissociation energy of the ionic bonds and entropic energy of the 
loaded network strands. High toughness of the hybrid gel is obtained when the alginate network is highly-crosslinked and the PAAm network is loosely-crosslinked [9,14-18], which mirrors the topologies known to maximize toughness in DN gels [19]. The ionic bonds that are pulled apart during the hybrid gel deformation are re-formed at zero-stress at a temperature-induced network recovery process to largely retrieve the mechanical properties of the virgin gel [9].

The unstable nature of the egg-box ionic crosslinks formed between the divalent cations and $\mathrm{G}$ groups on the alginate chains has been noted previously. In particular, a very large and rapid stress relaxation was reported in a $\mathrm{Ca}^{2+}$ ion crosslinked alginate single network. More than $90 \%$ of the initial stress relaxed when the ionic alginate gel was stretched and held at a set strain. The major mechanism accounting for the rapid stress relaxation was the unzipping of the ionic crosslinks. In many practical applications hydrogels are required to function under applied loads where stress relaxation and / or creep are undesirable. It is, therefore, important to determine the load stability and time-dependent mechanical properties of hybrid ionic / covalent tough gels.

The present study investigates the time-dependent behaviour of the ionic-covalent PAAm-alginate hybrid gel. We attempt to provide direct experimental evidence for the effect of the labile ionic crosslinks on the mechanical properties of the hybrid gel system over time. Rate-dependent toughness and stress relaxation tests were conducted on hybrid gel samples. We also characterize the mechanical strength and toughness of the hybrid gels prepared with various ionic and covalent crosslinker concentrations and swollen to equilibrium in water. Previous work studied the mechanical properties of the hybrid gels in the non-equilibrium, as-synthesized state. 


\section{Experimental}

\subsection{Materials}

The monomer of the covalently-crosslinked network was acrylamide (AAm, 40wt\% aqueous solution; Sigma-Aldrich). Potassium persulfate (KPS; Sigma-Aldrich) and N,N'methylenebisacrylamide (MBAA; Sigma-Aldrich) were used as radical initiator and crosslinking agent for the polymerization of PAAm gel, respectively. Sodium alginate from brown algae (Alg; Sigma-Aldrich) was used to prepare the ionically crosslinked network, and calcium chloride $\left(\mathrm{CaCl}_{2}\right.$; Sigma-Aldrich) was used as ionic crosslinker. All the chemical reagents were used as received. Deionized water was used in gel preparation.

\subsection{Hydrogel preparation}

Ionic-covalent PAAm-alginate hybrid gel. Two sets of sample gels were synthesized. In respective sets, the amount of $\mathrm{MBAA}$ or $\mathrm{CaCl}_{2}$ was varied systematically while the concentration of other chemical reagents remained constant. In set 1 , to prepare hybrid gels with different covalent crosslinking densities, $0.1 \mathrm{~mol} \%$ KPS and various amounts of MBAA $(0.01,0.05,0.1$, and $0.5 \mathrm{~mol} \%)$ were dissolved in $40 \mathrm{wt} \%$ aqueous AAm solution. The concentration of KPS and MBAA was with respect to the molar concentration of AAm. The alginate solution was made with $1.33 \mathrm{~g}$ alginate and $23 \mathrm{~g}$ deionized water. Two solutions were then blended and stirred for $2 \mathrm{~h}$ with final AAm and alginate concentrations at, respectively, $12 \mathrm{wt} \%$ and $4 \mathrm{wt} \%$. The gel solution was next bubbled by nitrogen for $40 \mathrm{~min}$ and then degassed in a vacuum environment for $2 \mathrm{~h}$ to remove dissolved oxygen, and then injected into a glass reaction mould consisting of two glass plates separated by a $2 \mathrm{~mm}$ thick silicon spacer. The mould was placed in a $60^{\circ} \mathrm{C}$ fanned oven for $6 \mathrm{~h}$ to finalize the polymerization. The resulting gels were called covalent PAAm-alginate gels until they were 
immersed in $2 \mathrm{wt} \% \mathrm{CaCl}_{2}$ solution for 5 days to ionically crosslink the alginate. In set 2 , MBAA and KPS concentration was fixed at 0.05 and 0.1 mol\% with respect to the molar concentration of AAm. All the gel preparation procedures were identical to set 1 until the covalent PAAm-alginate gels were obtained. Those gels were next immersed in $\mathrm{CaCl}_{2}$ solutions of different concentrations $(0.05,0.1,0.5$, and $2 \mathrm{wt} \%)$ to produce hybrid gels with various ionic crosslinking densities. In the following text, for simplicity, the hybrid gel is referred to by its characteristic MBAA or $\mathrm{Ca}^{2+}$ concentration. For example, in set 1 , the gel prepared with MBAA concentration at $0.05 \mathrm{~mol} \%$ is referred to as $0.05 \mathrm{~mol} \%$ gel. In set 2, if the gel was immersed in $2 \mathrm{wt} \% \mathrm{CaCl}_{2}$ solution, it was named as $2 \mathrm{wt} \%$ gel. Before any mechanical test was conducted, the hybrid gels were soaked in deionized water for 7 days to reach the swelling equilibrium.

Covalent PAAm-alginate gel. The same procedure introduced above was applied to prepare covalent PAAm-alginate gel. The only difference was that the gel was not immersed in $\mathrm{CaCl}_{2}$ solution, hence it was composed of non-ionically crosslinked alginate and covalently crosslinked PAAm network. In the present work, the covalent PAAm-alginate gel was prepared with identical chemical composition to the $0.05 \mathrm{~mol} \%$ hybrid gel, and it was used in the as-synthesized state in the stress relaxation test, since soaking in water would cause the loss of the uncrosslinked alginate from the gel.

PAAm gel. PAAm single network gel was prepared with $40 \mathrm{wt} \%$ acrylamide solution containing $0.1 \mathrm{~mol} \% \mathrm{KPS}$ and $0.05 \mathrm{~mol} \%$ MBAA with respect to the acrylamide. After the gel solution was bubbled with nitrogen, it was transferred into the same glass reaction mould used for hybrid gel synthesis. The mould was then placed in $60{ }^{\circ} \mathrm{C}$ fanned oven for $6 \mathrm{~h}$ to obtain PAAm single network gel. The as-synthesized PAAm gel was used directly in the 
stress relaxation test, since equilibrating in water would lead to excessive swelling of this loosely crosslinked gel.

\subsection{Tensile and fracture energy test}

All the mechanical measurements were carried out on an EZ-L mechanical tester (Shimadzu, Japan). Tensile tests were performed on dumbbell shaped samples with gauge length of $20 \mathrm{~mm}$ and width of $4 \mathrm{~mm}$. Strain and strain rate were determined by crosshead displacement. Standard rubber elasticity theory was fitted to the initial extension ratio $(\lambda)$ of stress $(\sigma)$-stretch curve to calculate shear modulus $(\mu)$ of each tested sample from [20]:

$\sigma=\mu\left(\lambda-\frac{1}{\lambda^{2}}\right)$

Fracture energy was obtained by the tearing test where the hybrid gel was cut into a rectangular shape $(50 \mathrm{~mm} \times 7.5 \mathrm{~mm})$ with a $20 \mathrm{~mm}$ long initial notch. The thickness of each sample was measured using a digital caliper. The fracture energy of a sample gel was calculated as:

$G=2 F_{\text {ave }} / w$

where $F_{\text {ave }}$ was average tearing force and $w$ was the thickness of the sample.

\subsection{Swelling test}

As-synthesized PAAm-alginate hybrid gels prepared with various MBAA or $\mathrm{Ca}^{2+}$ concentrations were soaked in excess deionized water to reach swelling equilibrium. The unreacted chemicals were removed in the process. The gels were then dried in a $60{ }^{\circ} \mathrm{C}$ fanned oven for a few days after which the dry mass of each gel was measured. The swelling ratio at equilibrium was calculated as the ratio of the equilibrium mass to the dry mass. 


\subsection{Stress relaxation test}

Stress relaxation tests were carried out on PAAm-alginate hybrid gel, covalent PAAmalginate gel, and PAAm single network gel to investigate their stress relaxation behaviours. In order to prevent water loss, the entire test was performed in an approximately $40 \mathrm{~cm}$ long polyethylene tube with an internal diameter of $4 \mathrm{~cm}$. Before the test, the tube was thoroughly rinsed with deionized water to create a moist inner environment. In the test, the gel was fixed at the tube bottom and attached to the upper crosshead of the tensile tester. Strain was determined from grip displacement. Unless otherwise specified, the grip displacement rate was $10 \mathrm{~mm} / \mathrm{min}$. The gel was first loaded to a set strain which was held for a particular period of time. The stress was recorded as a function of time. The sample was then unloaded.

\section{Results}

\subsection{Swelling ratios}

In the present study all sample gels were tested at the swelling equilibrium state in tensile and tearing tests. Table 1 indicates the swelling ratio of all sample gels after equilibration in deionized water. It is clear that the swelling ratio variation was very large among the gels prepared with various $\mathrm{Ca}^{2+}$ concentrations and decreased with increasing $\mathrm{Ca}^{2+}$ concentration. On the contrary, when the $\mathrm{Ca}^{2+}$ concentration was fixed, the gels prepared with different MBAA concentrations exhibited similar equilibrium swelling ratios. These results indicate that the ionic crosslinked alginate forms the tighter network of the two networks and controls the amount of hybrid gel swelling. The degree of swelling of the hybrid gels studied here is quite similar to those reported previously by Naficy et al. for hybrid gels that were prepared similarly and then swollen to equilibrium [18]. 
Table 1 Equilibrium swelling ratios of PAAm-alginate hybrid gels prepared with various MBAA and $\mathrm{Ca}^{2+}$ concentration.

\begin{tabular}{ccc}
\hline $\begin{array}{c}\text { MBAA concentration } \\
(\mathrm{mol} \%)\end{array}$ & $\begin{array}{c}\mathrm{CaCl}_{2} \text { concentration } \\
(\mathrm{wt} \%)\end{array}$ & Swelling ratio \\
\hline 0.01 & & 9.01 \\
0.05 & 2 & 9.43 \\
0.1 & & 9.37 \\
0.5 & 0.05 & 8.7 \\
\hline & 0.1 & 24.17 \\
0.05 & 0.5 & 15.88 \\
& 2 & 10.13 \\
& & 9.47 \\
\hline
\end{tabular}

\subsection{Tensile properties and fracture energy}

Figure 1 illustrates the effect of MBAA concentration on tensile properties of PAAmalginate hybrid gels. All samples were tested at a single strain rate of $10 \mathrm{~mm} / \mathrm{min}$. The effect of different strain rates on mechanical properties is considered in the next Section. Generally, the increase in MBAA concentration led to a noticeable decrease in tensile strength and elongation at break for the hybrid gels (Figure 1a). The measured tensile strength (Figure 1b) was $235 \pm 22$ and $277 \pm 13 \mathrm{kPa}$ for $0.5 \mathrm{~mol} \%$ and $0.1 \mathrm{~mol} \%$ gel, respectively, and it increased to $415 \pm 39$ and $429 \pm 48 \mathrm{kPa}$ when the MBAA concentration declined to 0.05 and $0.01 \mathrm{~mol} \%$. Similarly, the elongation at break $\left(\lambda_{\mathrm{b}}\right.$, Figure 1d) was less than 7 for $0.5 \mathrm{~mol} \%$ or $0.1 \mathrm{~mol} \%$ gel, while $\lambda_{\mathrm{b}}$ increased to above 12 if the MBAA concentration decreased to $0.05 \mathrm{~mol} \%$. It should be noted here that the sharpest increase in tensile strength and $\lambda_{\mathrm{b}}$ occurred when MBAA concentration was reduced from 0.1 to $0.05 \mathrm{~mol} \%$, whereas no significant enhancement in mechanical properties was observed between $0.05 \mathrm{~mol} \%$ and $0.01 \mathrm{~mol} \%$ gels. From these studies it is assumed that the critical value of MBAA concentration to enhance 
tensile properties of the hybrid gel is around $0.05 \mathrm{~mol} \%$. As shown in Figure 1c, MBAA concentration did not influence the shear modulus $(\mu)$ of the hybrid gel significantly. For a 50 fold change in covalent crosslinker concentration, there was no statistical difference in shear moduli. The PAAm-alginate hybrid gels studied here were prepared with $\mathrm{CaCl}_{2}$ concentration at $2 \mathrm{wt} \%$. The insensitivity of the hybrid gel modulus to covalent crosslinker concentration confirms that the covalent PAAm forms a loose network within the more tightly crosslinked ionic alginate network and the amount of covalent crosslinker has negligible influence on the initial stiffness of the overall hybrid gel.
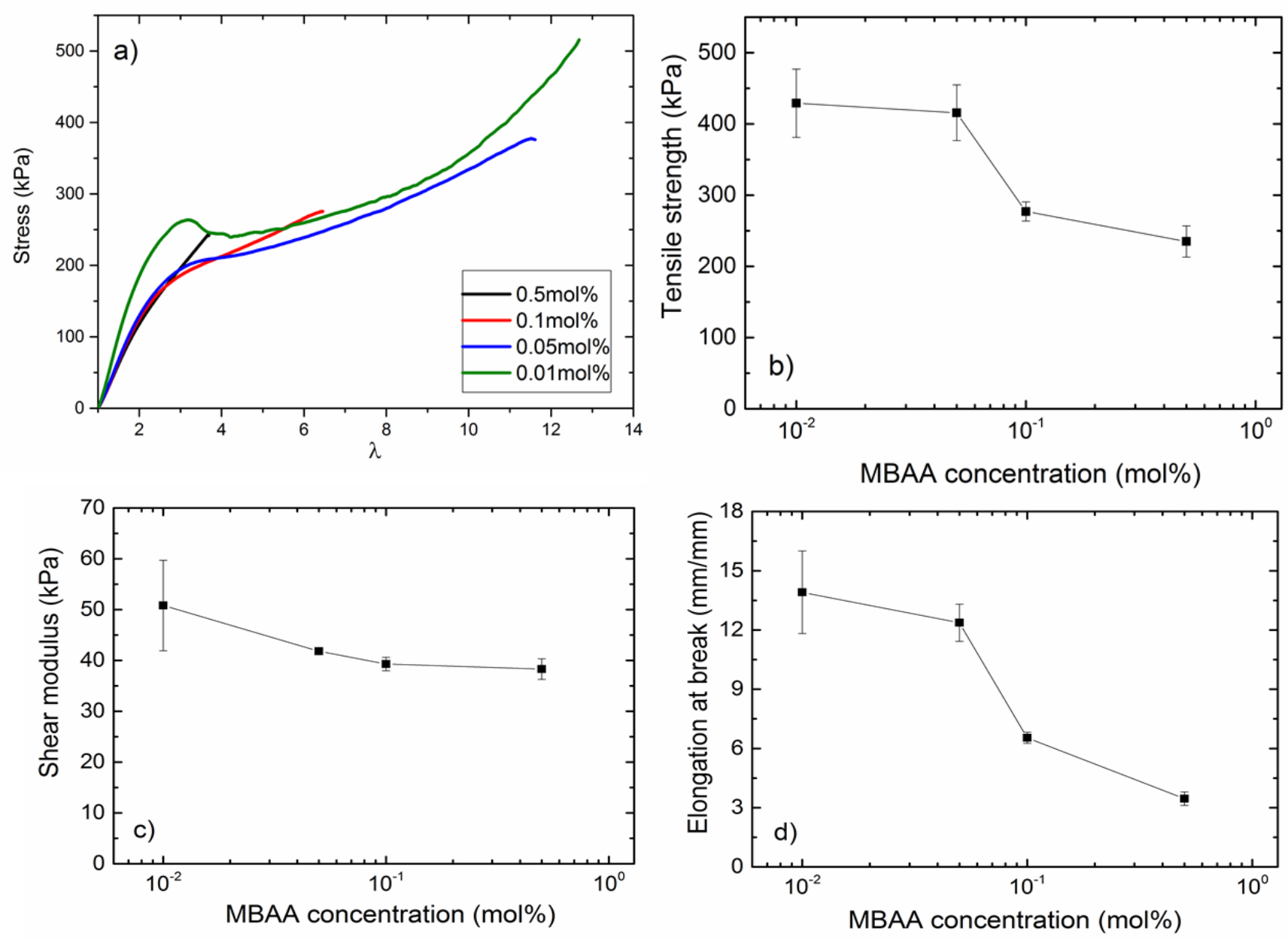
Figure 1 Effect of MBAA covalent crosslinker concentration on tensile properties of hybrid gels measured after swelling to equilibrium in deionized water. The $\mathrm{Ca}^{2+}$ concentration was held constant at $2 \mathrm{wt} \%$.

Figure 2 demonstrates tearing test results performed on PAAm-alginate hybrid gels that were prepared with various MBAA and $\mathrm{Ca}^{2+}$ concentrations. As shown in the figure, a plateau region where the load tends to be constant exists in all curves. This region is considered to be the stage where the pre-cut notch underwent stable propagation so that the average tearing force $F_{\text {ave }}$ was calculated from this region. Equation (2) was used to calculate fracture energies $(G)$ of all sample gels and the results are shown in Figure 3. It is clear that both MBAA and $\mathrm{Ca}^{2+}$ concentration have a large effect on the fracture toughness of PAAmalginate hybrid gels. Fracture energy was enhanced from $94 \pm 26$ to $1717 \pm 103 \mathrm{~J} / \mathrm{m}^{2}$ as MBAA concentration was reduced from 0.5 to $0.01 \mathrm{~mol} \%$, with $\mathrm{Ca}^{2+}$ concentration constant at $2 \mathrm{wt} \%$. In contrast, fracture energy remarkably increased from $151 \pm 49$ to $1280 \pm 56 \mathrm{~J} / \mathrm{m}^{2}$ with increasing $\mathrm{Ca}^{2+}$ concentration from 0.05 to $2 \mathrm{wt} \%$ for hybrid gels with constant covalent crosslinking at $0.05 \mathrm{~mol} \% \mathrm{MBAA}$. It should be noted that the peak toughness values are considerably lower than the maximum toughnesses reported previously for similar hybrid gels[9]. While slight differences in preparation methods may contribute to the lower toughness values reported here, the main contribution to the lower toughness is the higher swelling ratio (9-24) of the gels studied here in comparison to the previous work where the gels were tested in their as-synthesised state with swelling ratio of $\sim 7$. Increased swelling reduces the density of network chains thereby decreasing gel toughness as it has been previously shown that gel toughness is proportional to the density of network strands in the tight network [19]. 

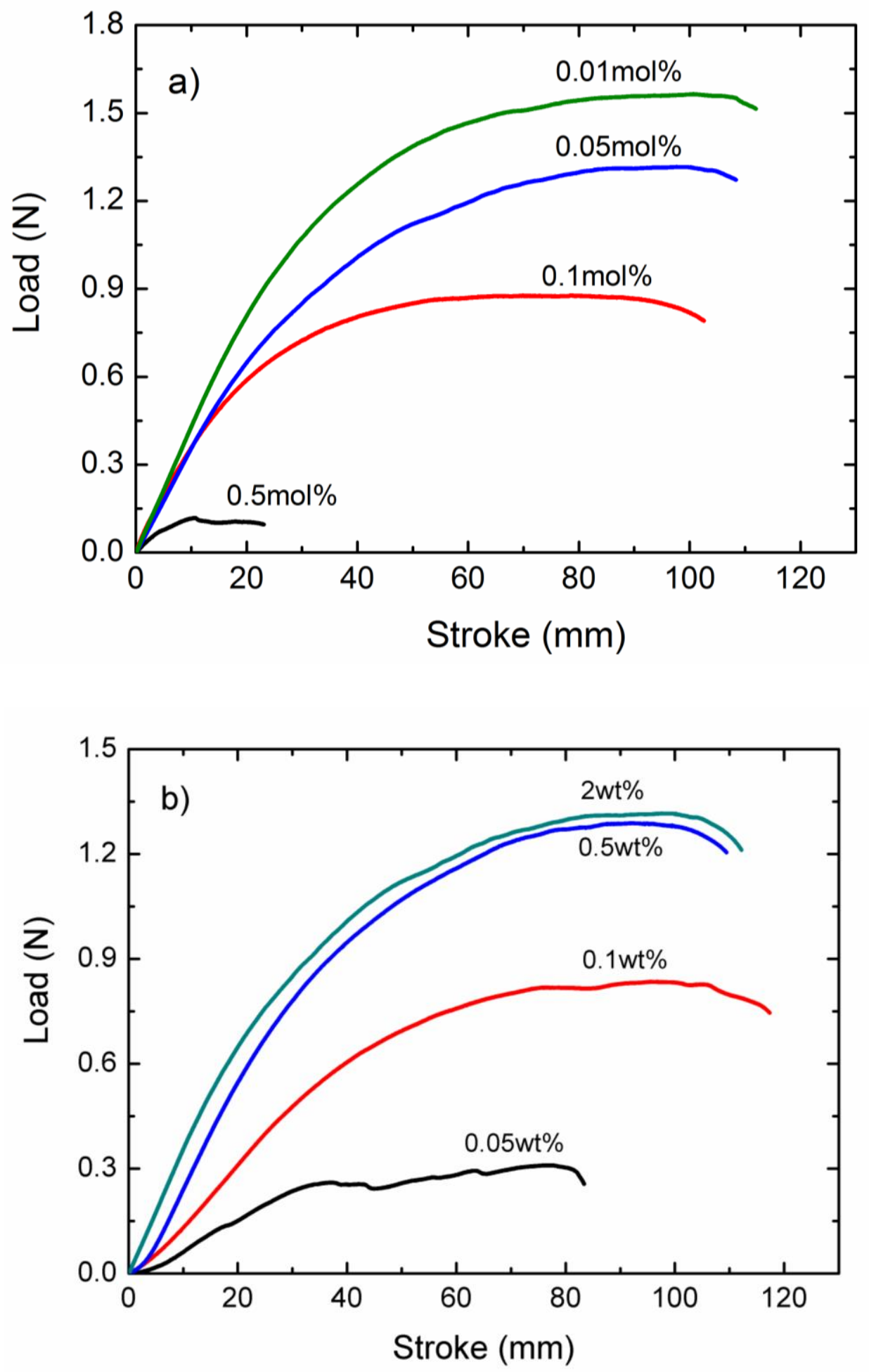

Figure 2 Tearing curves of PAAm-alginate hybrid gels prepared with various a) MBAA concentration and b) $\mathrm{Ca}^{2+}$ concentration. The $\mathrm{Ca}^{2+}$ concentration was 2 wt $\%$ in (a) and MBAA concentration was $0.05 \mathrm{~mol} \%$ in (b). 

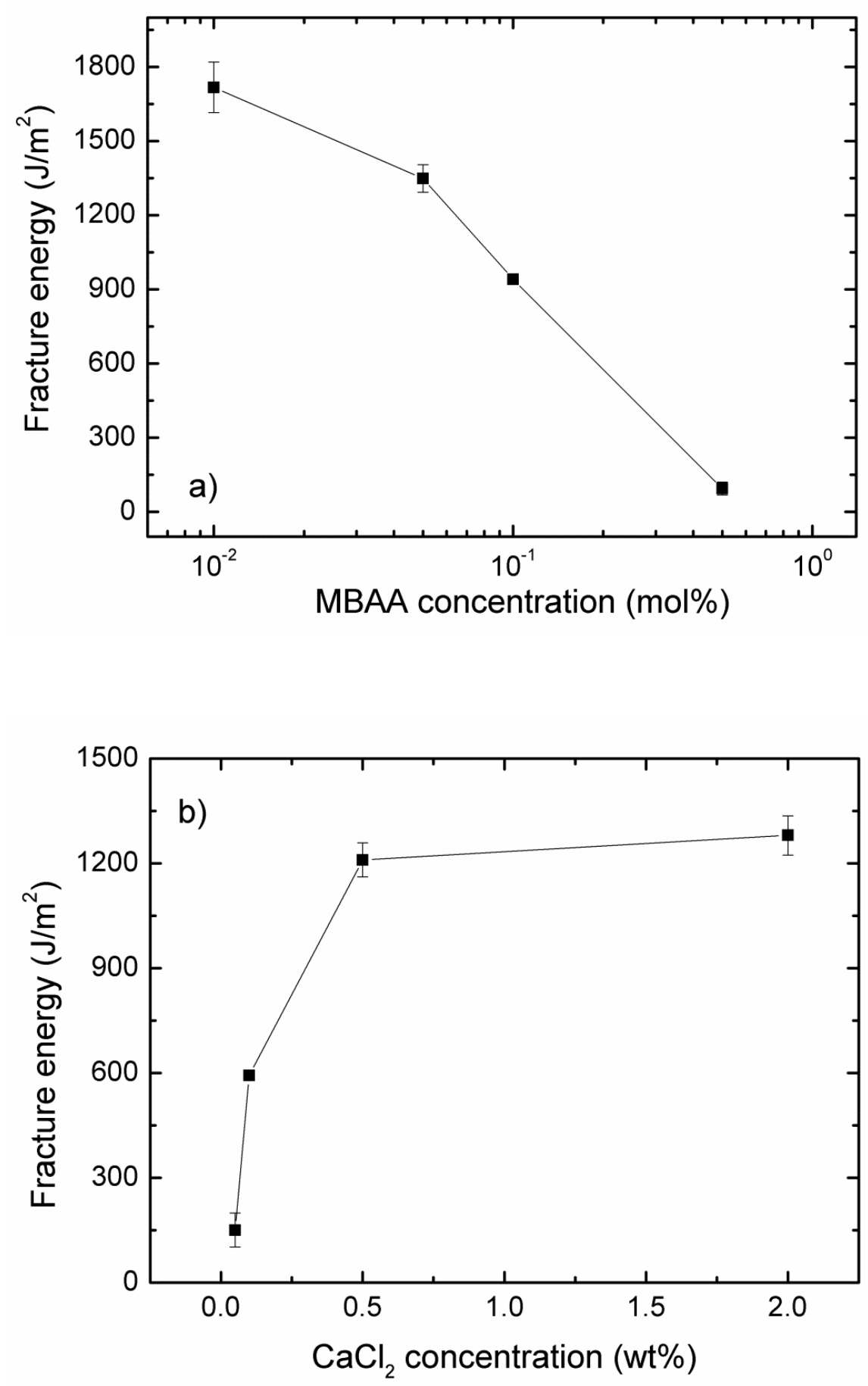

Figure 3 Effect of a) MBAA covalent crosslinker concentration $\left(\mathrm{Ca}^{2+}\right.$ ionic crosslinker concentration fixed at $2 \mathrm{wt} \%$ ) and b) $\mathrm{Ca}^{2+}$ ionic crosslinker concentration (covalent MBAA crosslinker concentration fixed at $0.05 \mathrm{~mol} \%$ ) on fracture energy of PAAm-alginate hybrid gels. 
The results shown in Figures 1-3 clearly illustrate the effect of MBAA and $\mathrm{Ca}^{2+}$ concentration on tensile properties and fracture energy of the fully swollen PAAm-alginate hybrid gel. The observations mirror the findings for double network gels where toughness enhancement is produced by the presence of a tight network that is reinforced with a much larger quantity of a loose network [19,21]. In the hybrid gels studied here (and previously in $[9,18]$ ), a densely-crosslinked alginate and a loosely-crosslinked PAAm network are necessary conditions for toughness enhancement. Suo et al. have reported the effect of both $\mathrm{Ca}^{2+}$ and MBAA concentration on tensile strength, elastic modulus, and fracture energy of PAAm-alginate hybrid gels prepared in a one-pot synthesis procedure and tested assynthesized (i.e. not swollen to equilibrium). The fracture energy was reported to peak at an intermediate concentration for both covalent and ionic crosslinker. The peak fracture energies were $8,000-9,000 \mathrm{~J} / \mathrm{m}^{2}$ or $4-5$ times higher than the maximum fracture energies reported in the present study [9]. These differences in fracture energy likely result from the different preparation conditions and the fact that the hybrid hydrogels studied here were fully swollen in water.

For interpreting the mechanical properties of PAAm-alginate hybrid gels, the fracture model accounting for the damage process of covalent Double Network hydrogels (DN gel) can be utilized here, since both DN and the hybrid gel systems exhibit enhanced mechanical toughness with the combination of a tight and a loose network. In a DN gel, very large fracture toughness is derived from energy dissipation by irreversible covalent bond (sacrificial bonds) scission of the $1^{\text {st }}$ (tight) network. The multiple damage zones that develop in the $1^{\text {st }}$ network are held together by the longer and more prevalent $2^{\text {nd }}$ network chains so that catastrophic failure is prevented. Therefore, the remarkable toughness of DN gels is obtained when the $1^{\text {st }}$ network is highly-crosslinked and the $2^{\text {nd }}$ network is loosely crosslinked [19,21-24]. The former condition guarantees a large sum of short polymer strands 
whose entropic energy is dissipated during crack development. The latter one ensures $2^{\text {nd }}$ network chains to be long and elastic enough to bridge $1^{\text {st }}$ network damage zones.

In ionic-covalent PAAm-alginate hybrid gel, guluronic acid (G group) from different alginate chains are connected by $\mathrm{Ca}^{2+}$ ions, forming ionic crosslinks which are described by a widely-accepted 'egg-box' model [12,13]. When the ionically crosslinked alginate network is subject to stress, the crosslinks are pulled apart in a process known as an 'unzipping' effect that ultimately leads to the unloading of the network strands [14,15]. For single alginate networks, such an unzipping will lead to macroscopic failure because of damage localization. In contrast, the hybrid gel studied here has an interpenetrating network (IPN) with mutual network entanglement. It has been suggested that alginate chains are evenly dispersed within the PAAm network [9]. As a consequence, when a hybrid gel is stressed above a certain level, the unzipping will lead to damage zones that are bridged and stabilised by PAAm network strands $[9,16]$. The more ionically bonded alginate network strands that are unzipped, the more energy is dissipated, leading to higher toughness. Increase in $\mathrm{Ca}^{2+}$ concentration increases the number of ionic crosslinks to be broken per unit volume, and a decrease in MBAA concentration produces longer PAAm chains that are better able to stabilise unzipped alginate chains. Hence, tougher hydrogel systems are obtained when higher concentration of $\mathrm{Ca}^{2+}$ is used along with lower concentration of MBAA. Similarly for DN gels, these two conditions were necessary for obtaining a tough hybrid gel. 


\subsection{Rate dependence of PAAm-alginate hybrid gels}

Tearing tests were conducted with various crosshead displacement velocities to investigate rate-dependence of toughness in hybrid gels. The $0.05 \mathrm{~mol} \% \mathrm{MBAA} / 2 \mathrm{wt} \% \mathrm{Ca}^{2+}$ gel was chosen as the sample gel for these studies because of their high toughness and consistent properties. As shown clearly in Figure 4, the gel underwent a steady tearing stage at each test rate before the occurrence of ultimate rupture. The dependence of fracture energy $(G)$ on test rates is shown in Figure 4 (b). Unlike in DN gels where toughness is almost rate independent [25,26], the hybrid gel in the present work exhibited a noticeable ratedependence. The tested fracture energy increased from 1157 to $1748 \mathrm{~J} / \mathrm{m}^{2}$ or $\sim 50 \%$ as crosshead velocity increased from 1 to $500 \mathrm{~mm} / \mathrm{min}$. The rate-dependent fracture toughness for ionically-crosslinked alginate single network gels has also been reported previously, where fracture propagates via the progressive unzipping of the ionically crosslinked G blocks on alginate chains resulting in pull out of the free chains against the solvent. The unzipping effect was shown to be thermally activated and stress-aided. Both plastic unzipping and viscous pulling-out are thought to be "rate-strengthening" mechanisms [14,15]. Since the dissociation of ionically crosslinked alginate network is the main source of energy dissipation in the hybrid hydrogel, the same rate-dependence of toughness observed in the hybrid gels is likely related to the same processes identified in the single alginate network. 

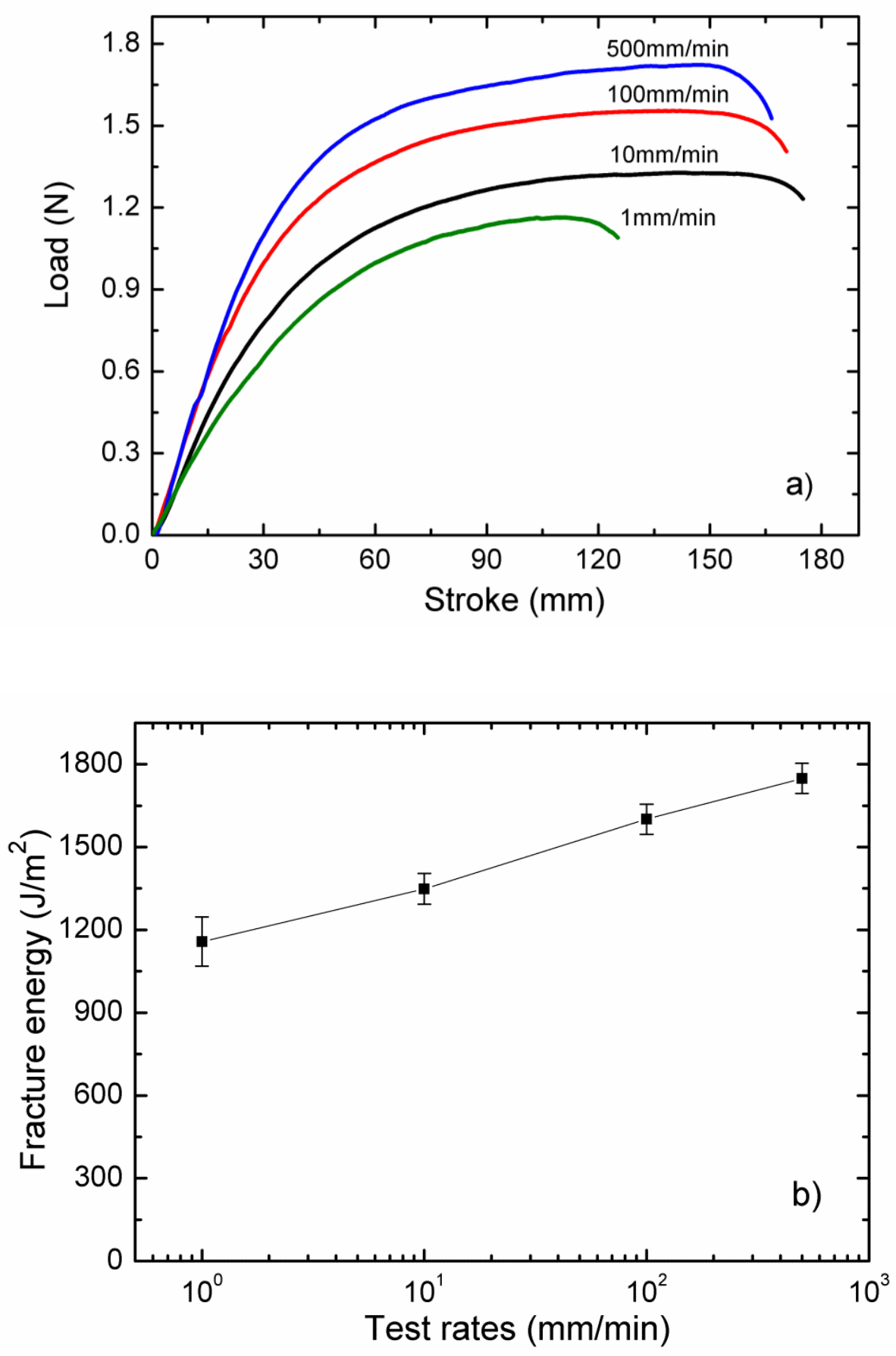

Figure 4 Test rates effect on a) tearing force and b) fracture energy of hybrid gels. 


\subsection{Stress relaxation}

Stress relaxation tests were performed on an ionic-covalent PAAm-alginate hybrid gel $\left(0.05 \mathrm{~mol} \% \mathrm{MBAA}\right.$ and $\left.2 \mathrm{wt} \% \mathrm{Ca}^{2+}\right)$ to evaluate the stability of the ionic crosslinks under load. For comparison, stress relaxation tests were also performed on a non-ionically crosslinked PAAm-alginate gel and a PAAm single network gel with both tested in their assynthesized state. The former sample had the same chemical composition as the $0.05 \mathrm{~mol} \%$ MBAA / $2 \mathrm{wt} \% \mathrm{Ca}^{2+}$ hybrid gel but was prepared without soaking in $\mathrm{Ca}^{2+}$ ionic crosslinking solution. This sample is denoted NI-hybrid to signify that it was not ionically crosslinked. The latter sample was prepared from the identical acrylamide solution used in $0.05 \mathrm{~mol} \%$ MBAA / $2 \mathrm{wt} \% \mathrm{Ca}^{2+}$ hybrid gel synthesis but without addition of alginate and with no treatment with $\mathrm{Ca}^{2+}$. The stress-extension curves of the tests are shown in Figures 5 and 6 , where the insets illustrate the stress relaxation of each gel as a function of time after the loading period. Comparing Figures 5 and 6 it can be seen that the loading stress to $\lambda=2$ varied considerably, which reflects differences in crosslink density and water content. The latter decreased as hybrid gel ( $89 \mathrm{wt} \%)$, NI- hybrid (83 wt $\%$ ) and PAAm single network (60 wt $\%$ ).

As shown in Figure 5, a very fast and substantial stress relaxation occurred in the hybrid gel after it was loaded to $\lambda=2$ and held at this strain for $1 \mathrm{~h}$. Approximately half of the maximum loading stress was relaxed throughout the holding period. Meanwhile, the relaxation rate was very rapid during the initial 600 seconds. By contrast, as shown in Figure 6, both the NI-hybrid gel and the PAAm single network gel showed comparatively smaller stress relaxation of $\sim 10 \%$ or less during the $1 \mathrm{~h}$ holding period after extension to $\lambda=2$. It is important to note that all three samples were tested in air but in an enclosed environment to reduce evaporation. Consequently, any stress relaxation observed here is due to the structural deformation of the networks. Clearly, the ionically crosslinked alginate network suffers a 
much greater degree of stress relaxation than the covalent network shows both in the presence or absence of non-crosslinked alginate. The three networks compared in Figures 5 and 6 also show significantly different stresses during loading. Crosslinking the alginate network in the hybrid gel (Fig. 5) increases the stress to reach an extension of $\lambda=2$ by $\sim 35$ times compared to the NI-hybrid gel because of the presence of the tightly crosslinked alginate network. The PAAm single network is less stiff than the hybrid gel because of the absence of the ionic network, but is stiffer than the NI-hybrid because of a reduced swelling ratio in the assynthesized state (the pre-gel solution was not diluted with alginate solution).

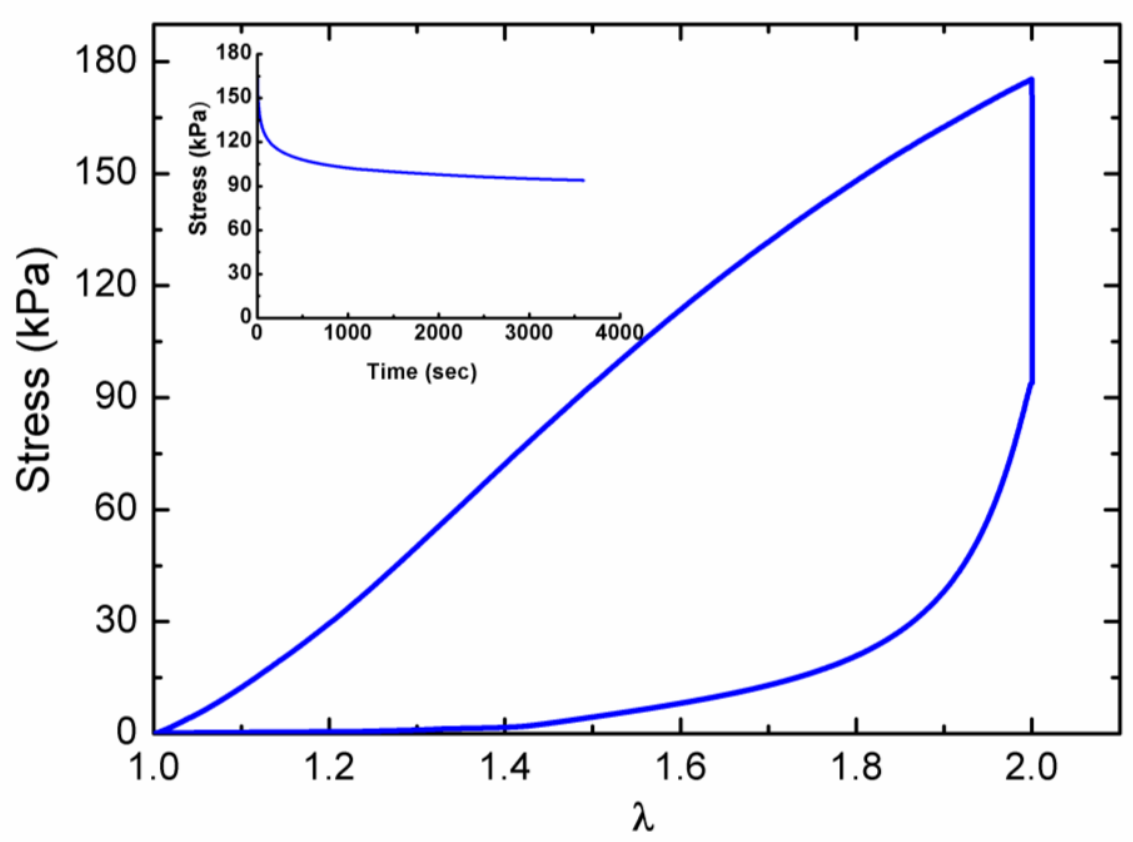

Figure 5 Stress relaxation test for fully swollen PAAm-alginate hybrid gel. MBAA: 0.05 mol\%, $\mathrm{Ca}^{2+}: 2 \mathrm{wt} \%$. Inset shows stress vs time at $\lambda=2$. 

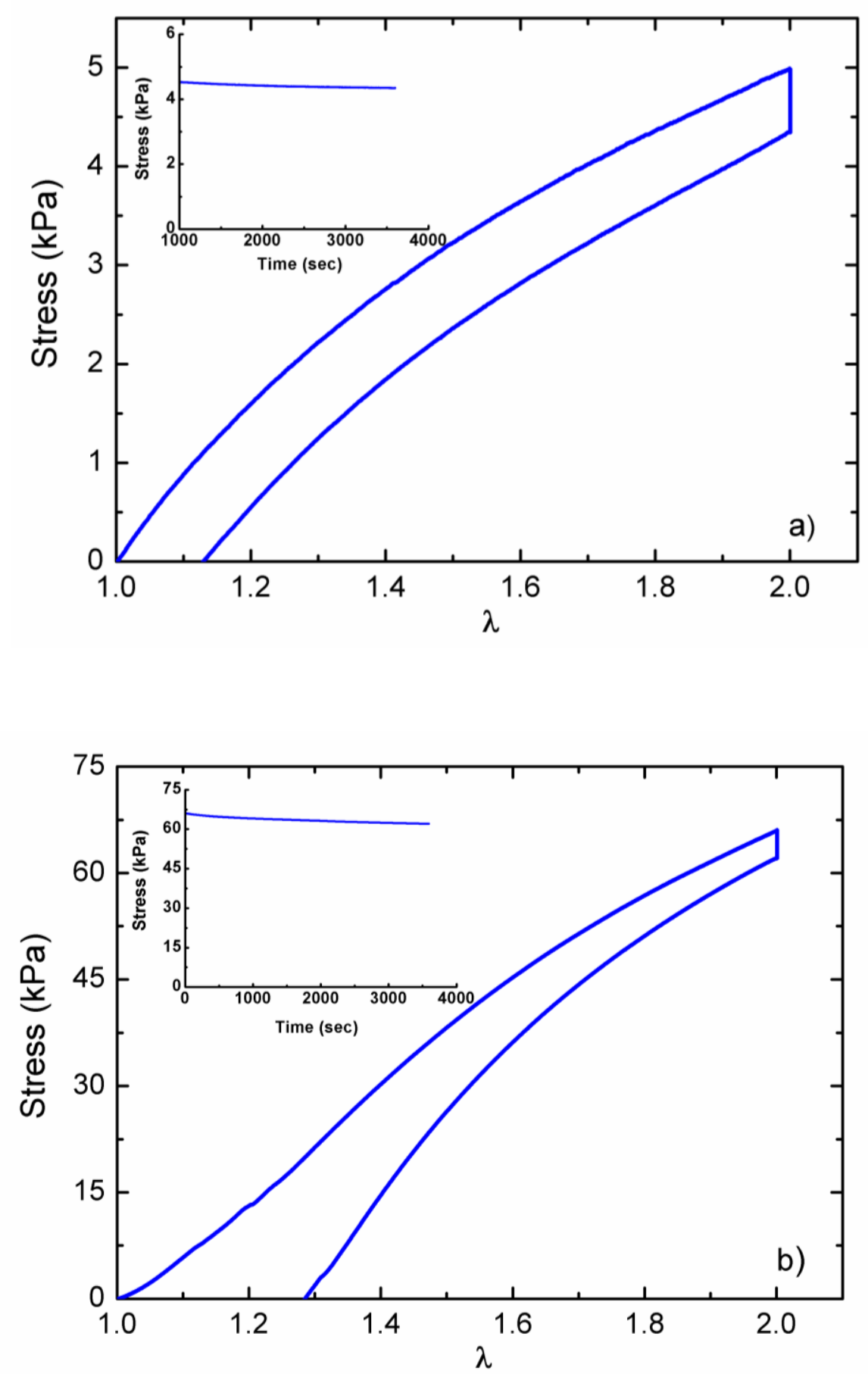

Figure 6 Stress relaxation test for a) non-ionically crosslinked PAAm-alginate hybrid gel and b) covalent PAAm single network gel. Inset shows stress relaxation during the constant strain hold period, following loading and preceding unloading. 
A more detailed study on the relaxation behaviour of the hybrid gels was conducted through relaxation tests on the hybrid gel by using three different crosshead velocities applied during loading. The sample gel was loaded to $\lambda$ of 2 at $1 \mathrm{~mm} / \mathrm{min}, 10 \mathrm{~mm} / \mathrm{min}$, and 100 $\mathrm{mm} / \mathrm{min}$, respectively, and the strain was held for $20 \mathrm{~min}$ to allow the stress to relax. As shown in Figure 7a, the gel exhibited rate-dependence during loading where the stress needed to achieve a given extension decreased at slower loading rates. This observation suggests that the level of crosslinking decreased during loading, especially at the slower loading rates. Figure $7 \mathrm{~b}$ illustrates how the stress relaxed as a function of time in each of the three tests. It is seen that by increasing the loading rate the relaxation started from a significantly higher stress than for samples tested at the slower loading rates, and the final stress levels were similar for all three loading rates. The unloading curves after the stress relaxation period (Figure 7a) were very similar regardless of initial loading rate and the different amounts of stress relaxation. This observation suggests that the networks reached a similar state of crosslinking following the loading and hold periods. 

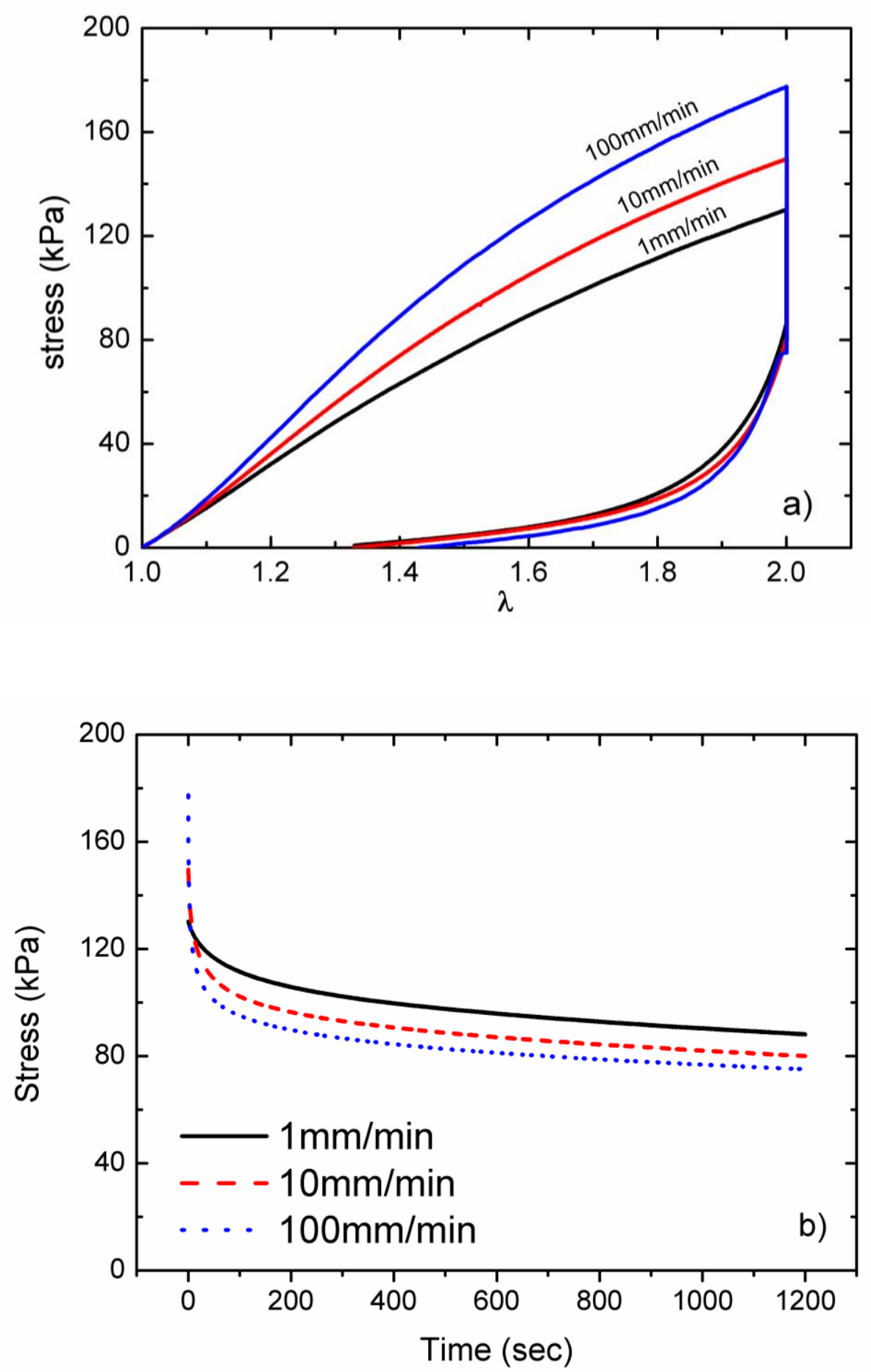

Figure 7 Loading, stress relaxation and unloading tests performed on the 0.05 mol\% MBAA / $2 \mathrm{wt} . \% \mathrm{Ca}^{2+}$ hybrid gel at three different loading rates, as indicated. a) Stress-stretch curves and $b$ ) stress relaxation with time at a constant extension of $\lambda=2$ following the three different loading tests. 
The Maxwell model [20,27] is often used to mathematically describe the stress relaxation of polymers and is applied here to the hybrid gels. This model takes the form of a single exponential as shown in equation (3) where $\sigma$ is stress at time $t, \sigma(0)$ is the initial stress, and $\tau$ represents the relaxation time. The larger the value of $\tau$, the more elasticity the material exhibits and the slower is the rate of stress relaxation.

$$
\sigma(t)=\sigma(0) e^{-t / \tau}
$$

Attempts to apply the simple Maxwell model to the stress relaxation data were unsuccessful (Figure 8). The new approach was to find the best fit to the experimental data from the intermediate loading rate $(10 \mathrm{~mm} / \mathrm{min})$ in Figure $7 \mathrm{~b}$ and then apply the fitted relaxation time to the data obtained from the two other loading rates. As shown in Figure 8, the Maxwell model was unable to adequately describe the relaxation over the full relaxation period. The longer time relaxation was best captured with a relaxation time of 530 seconds (Figure 8a) but the rapid initial relaxation was best modelled with a relaxation time of 25 seconds (Figure 8b). No single relaxation time was able to satisfactorily fit the entire relaxation process.

Based on these observations, a double exponential model in the form of equation (4) was proposed. Here, $\sigma_{\mathrm{o}}$ was designed as a constant for fitting purposes, which was then adjusted to approximately match the experimentally measured stress obtained at the end of the loading period. $\sigma(t), t$, and $\tau$ have the same physical meanings as in Maxwell model.

$$
\sigma(t)=\sigma_{\mathrm{o}}+\mathrm{A}_{1} \mathrm{e}^{-t / \tau_{1}}+\mathrm{A}_{2} \mathrm{e}^{-t / \tau_{2}}
$$



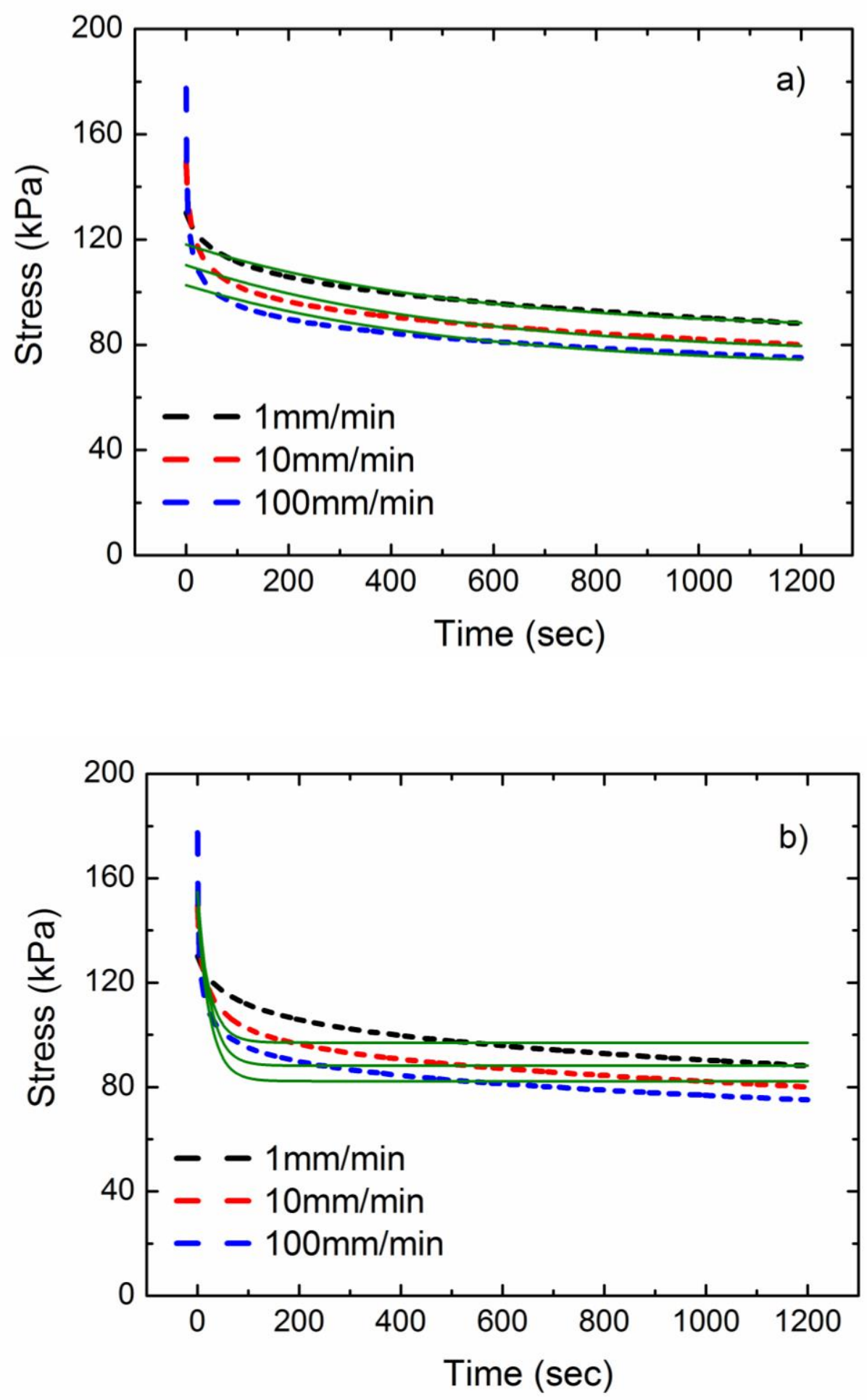

Figure 8 Experimental data (dashed lines) and Maxwell model exponential fits (solid lines) to stress relaxation of the hybrid gels following loading to an extension ratio of 2 at three different rates and modelled using a single relaxation time of a) 530 seconds; and b) 25 seconds. 
Equation (4) was first fitted to the stress relaxation curve obtained from the $10 \mathrm{~mm} / \mathrm{min}$ loading test to obtain model parameters. Since the gels used in the three tests were the same, the relaxation times $\left(\tau_{1}\right.$ and $\tau_{2}$ ) obtained from the $10 \mathrm{~mm} / \mathrm{min}$ loading test were determined by fitting and then applied to the results obtained from the $1 \mathrm{~mm} / \mathrm{min}$ and $100 \mathrm{~mm} / \mathrm{min}$ tests. Figure 9a illustrates the relaxation curve fits using equation (4). To have a clearer demonstration on the fitting quality, the stress was re-plotted against logarithm of time in Figure $9 \mathrm{~b}$. It is shown that the proposed double exponential model was able to provide more satisfactory fits to the entire stress decay of the hybrid gel as compared with Maxwell's single relaxation model. The fitted model parameters are shown in Table 2. With increasing the first-loading rate, the values of $\mathrm{A}_{1}$ decreased and $\mathrm{A}_{2}$ increased.

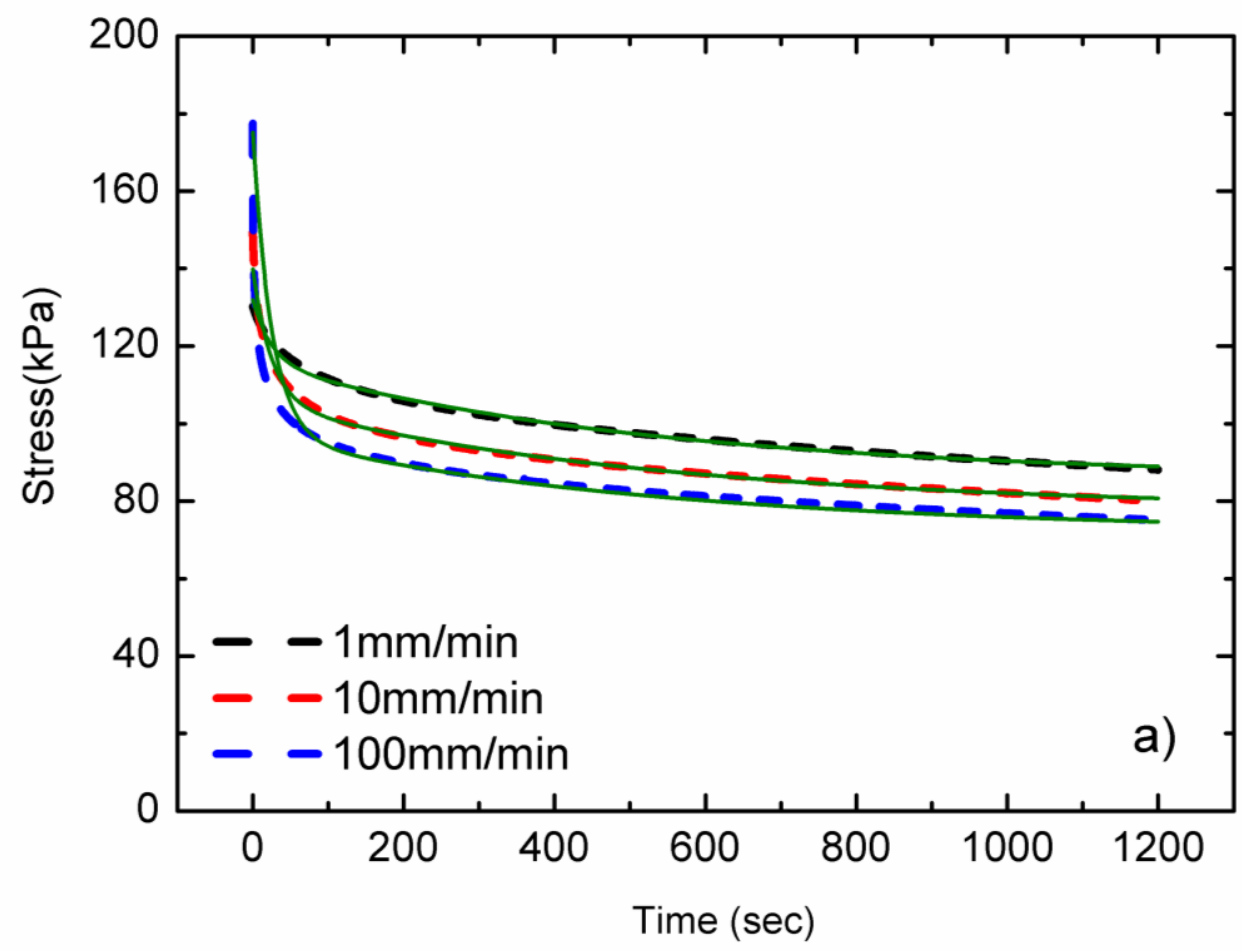




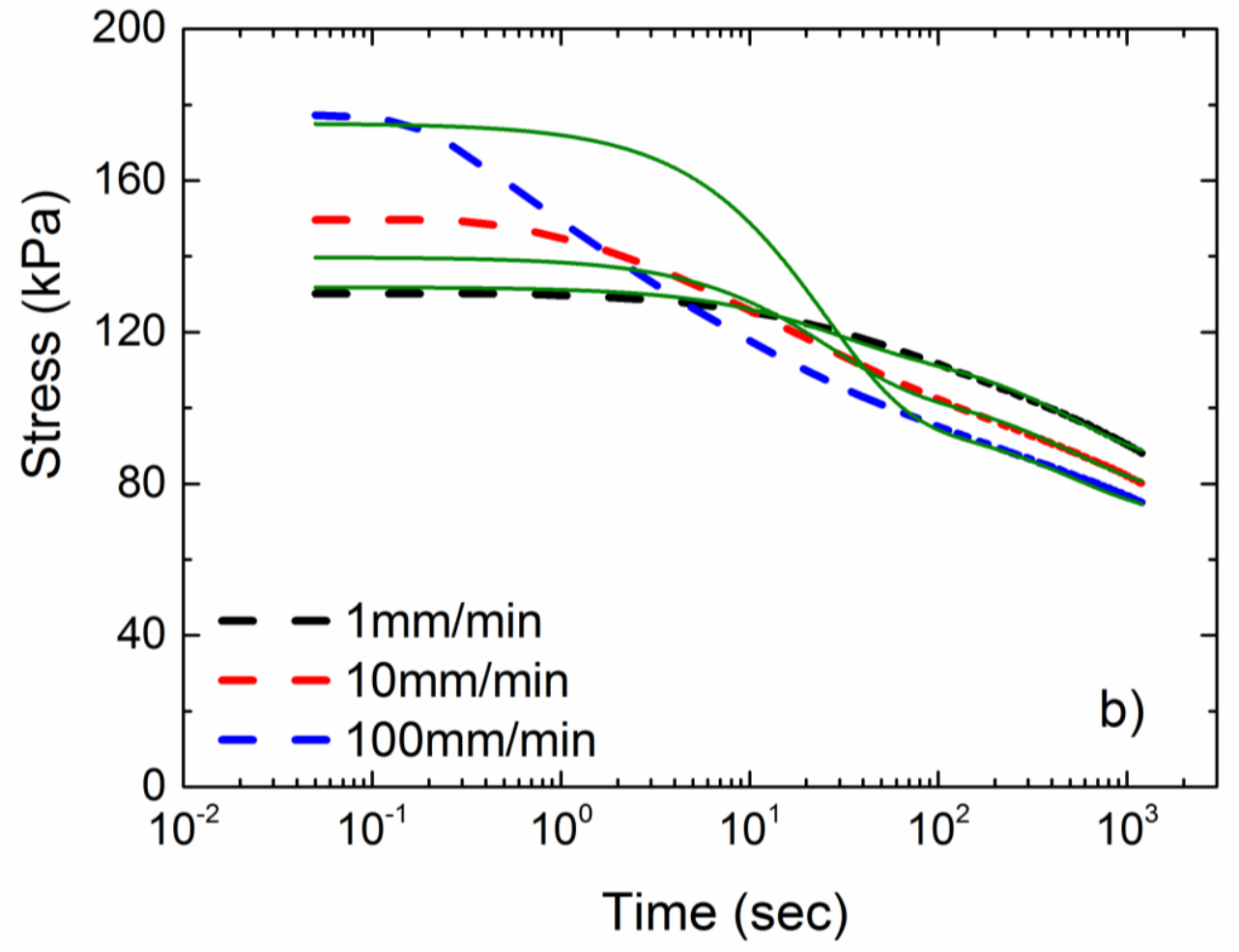

Figure 9 The double exponential fits to stress relaxation of the hybrid gels after loading to a given strain at three different rates. The dashed lines are measured stress decay as a function of time and the green solid lines are double exponential fits. The stress is plotted against linear time in a) and log time in b) respectively.

Table 2 Fitted parameters of the double exponential to the stress relaxation of the hybrid gels.

\begin{tabular}{cccc}
\hline First-loading rate & $1 \mathrm{~mm} / \mathrm{min}$ & $10 \mathrm{~mm} / \mathrm{min}$ & $100 \mathrm{~mm} / \mathrm{min}$ \\
\hline$\sigma_{\mathrm{o}}(\mathrm{kPa})$ & 86 & 78 & 72 \\
$\mathrm{~A} 1$ & 30 & 28 & 25 \\
$\tau_{1}(\mathrm{sec})$ & 530 & 530 & 530 \\
$\mathrm{~A} 2$ & 16 & 34 & 78 \\
$\tau_{2}(\mathrm{sec})$ & 25 & 25 & 25 \\
\hline
\end{tabular}


The satisfactory fits using a double exponential Maxwell model of stress relaxation in the hybrid gel indicates that the process can be described by the combined role of two mechanisms involving a slow mode represented by $\mathrm{A}_{1}$ and $\tau_{1}(530 \mathrm{sec})$ and a fast mode represented by $\mathrm{A}_{2}$ and $\tau_{2}(25 \mathrm{sec})$. With an increase in the loading rate, the values of $\mathrm{A}_{1}$ (slow mode) decreased while $A_{2}$ (fast mode) increased. This trend indicates that in the faster loading test, less relaxation could occur during the loading process so that during the hold period the stress relaxation was dominated by the fast mode. In other words, when a gel was loaded to the set strain at a faster rate, there was less time for the stress to relax during the loading process. Hence, its stress relaxation behaviour was more dependent on the fast mode which is reflected by the increase in $\mathrm{A}_{2}$.

\section{Discussion}

The experimental results shown above in section 3.3 and 3.4 suggest that ionic-covalent PAAm-alginate hybrid gel has a very distinctive stress relaxation behaviour compared to conventional covalently-crosslinked hydrogels. In addition, and in contrast to the DN gels, the hybrid gel in the present work exhibits rate-dependent toughness. Both of these characteristics are considered to be associated with the ionic crosslinks in the gel network, as is the recoverable nature of the load-induced network damage.

Previous investigations have compared the stress relaxation of ionically-crosslinked and covalently-crosslinked alginate single network hydrogels $[28,29]$. The ionically crosslinked alginate displayed very large and fast stress relaxation of more than $90 \%$ of the maximum loading stress. By contrast, the covalently crosslinked alginate gel showed a much smaller stress relaxation [28]. These studies clearly implicate the labile ionic crosslinks in the stress relaxation process and the relaxation mechanism has been ascribed to the fracture/re- 
formation of ionic crosslinks [28,29] which results in the internal re-construction of the alginate network.

In the present study it has been shown that the dissociation of ionic bonds under load is time dependent. When the hybrid gel is stretched at a slow rate a greater number of ionic bonds can be cleaved during the loading process. The ionic crosslinking density is thereby reduced when compared to faster loading. The gel deformed slowly became much softer, and less stress was needed to deform the gel to a set strain. The same process occurs in the high stress region ahead of a crack tip. Slow rates of crack propagation provide more time for ionic crosslinks to dissociate in the process zone ahead of the crack tip. The reduced ionic crosslink density then translates to a lower toughness, since there remain fewer strands to unload and dissipate energy. On the contrary, if the gel was deformed at a faster velocity, fewer ionic bonds were pulled apart because of insufficient time. Accordingly, the density of short polymer strands remained higher, leading to the higher toughness.

The stress relaxation tests could not be fitted to a single exponential relaxation process, but a double exponential provided satisfactory fits to the experimental data. The two exponential terms had quite different time constants and likely reflect two different molecular mechanisms. Ionic bond dissociation reduces crosslink density and network stiffness and thereby decreases stress in the network under constant strain. Secondly, molecular strand rearrangement, perhaps facilitated by loss of crosslinks, could also allow stress reductions. Further work is required to properly identify these molecular processes and their time dependencies. 


\section{Conclusions}

Two series of ionic-covalent PAAm-alginate hybrid gels were prepared with varying concentrations of covalent crosslinker (MBAA) and ionic crosslinker $\left(\mathrm{CaCl}_{2}\right)$, respectively. Mechanical properties of obtained gels were found to mirror previous observations made for DN gels with higher toughness, strength and elongation at break afforded by a tight network supported by a larger fraction of loose network. Here, the tight network is produced by the ionically crosslinked alginate and the mechanical properties were improved by increasing $\mathrm{Ca}^{2+}$ concentration used to crosslink the alginate. The loose network was provided by the covalently crosslinked PAAm and mechanical properties were improved by using smaller amounts of MBAA crosslinker. The shear modulus and equilibrium swelling ratios were dominated by the degree of ionic crosslinking and were little affected by the amount of covalent crosslinking. These results support the previous finding of Suo et al [9] regarding the optimum network topologies for toughening but also demonstrate that substantial reductions in toughness occur in the equilibrium swollen state compared with the lessswollen, as-synthesised state. The highest toughness report here for fully swollen networks were of the order of $1800 \mathrm{~J} / \mathrm{m}^{2}$, which is amongst the toughest gels reported.

Stress relaxation tests showed very large stress relaxation in the ionically-crosslinked hybrid gel. In contrast little stress relaxation occurred in covalent gel systems. A double exponential model was used to mathematically describe the stress relaxation behaviours of the hybrid gels loaded and held to a set strain at three different straining rates. The model consists of a fast and a slow mode by which the overall relaxation behaviours of the hybrid gel are determined. Both rate-dependent toughness and stress relaxation behaviour of the hybrid gel were associated with the time dependent dissociation of the ionic crosslinks. While the labile nature of the ionic crosslinks affords high toughness and recoverability of 
properties following deformation, the very large stress relaxations also present practical difficulties. In situations where load is to be applied for long periods, the gels will suffer stress loss or creep which would be undesirable in applications, such as for artificial muscles.

\section{References}

(1) Calvert P. Advanced Materials 2009;21(7):743-756.

(2) Deligkaris K, Tadele TS, Olthuis W and van den Berg A. Sensors and Actuators BChemical 2010;147(2):765-774.

(3) Kopecek J and Yang JY. Polymer International 2007;56(9):1078-1098.

(4) Gong JP, Katsuyama Y, Kurokawa $\mathrm{T}$ and Osada Y. Advanced Materials 2003;15(14):1155-1158.

(5) Haraguchi K and Takehisa T. Advanced Materials 2002;14(16):1120-1124.

(6) Ito K. Polymer Journal 2007;39(6):489-499.

(7) Naficy S, Brown HR, Razal JM, Spinks GM and Whitten PG. Aust. J. Chem. 2011;64(8):1007-1025.

(8) Bakarich SE, Pidcock GC, Balding P, Stevens L, Calvert P and Panhuis MIH. Soft Matter 2012;8(39):9985-9988.

(9) Sun JY, Zhao XH, Illeperuma WRK, Chaudhuri O, Oh KH, Mooney DJ, Vlassak JJ and Suo ZG. Nature 2012;489(7414):133-136.

(10) Augst AD, Kong HJ and Mooney DJ. Macromolecular Bioscience 2006;6(8):623-633.

(11) Lee KY and Mooney DJ. Progress in Polymer Science 2012;37(1):106-126.

(12) Grant GT, Morris ER, Rees DA, Smith PJC and Thom D. Febs Letters 1973;32(1):195-198.

(13) Braccini I and Perez S. Biomacromolecules 2001;2(4):1089-1096.

(14) Baumberger T and Ronsin O. Journal of Chemical Physics 2009;130(6):061102.

(15) Baumberger T and Ronsin O. Biomacromolecules 2010;11(6):1571-1578. 
(16) Yang CH, Wang MX, Haider H, Yang JH, Sun JY, Chen YM, Zhou JX and Suo ZG. Acs Applied Materials \& Interfaces 2013;5(21):10418-10422.

(17) Li JY, Illeperuma WBK, Suo ZG and Vlassak JJ. Acs Macro Letters 2014;3(6):520523.

(18) Naficy S, Kawakami S, Sadegholvaad S, Wakisaka M and Spinks GM. Journal of Applied Polymer Science 2013;130(4):2504-2513.

(19) Xin H, Saricilar SZ, Brown HR, Whitten PG and Spinks GM. Macromolecules 2013;46(16):6613-6620.

(20) Rubinstein M and Colby RH Polymer Physics; Oxford University Press: Oxford, 2006.

(21) Gong JP. Soft Matter 2010;6(12):2583-2590.

(22) Brown HR. Macromolecules 2007;40(10):3815-3818.

(23) Tanaka Y. Europhysics Letters 2007;78(5):56005.

(24) Gong JP. Science 2014;344(6180):161-162.

(25) Tanaka Y, Kuwabara R, Na Y-H, Kurokawa T, Gong JP and Osada Y. Journal of Physical Chemistry B 2005;109(23):11559-11562.

(26) Webber RE, Creton C, Brown HR and Gong JP. Macromolecules 2007;40(8):29192927.

(27) Bueche FJ Physical Properties of Polymers; Wiley Interscience: New York, 1962.

(28) Zhao XH, Huebsch N, Mooney DJ and Suo ZG. Journal of Applied Physics 2010;107(6).

(29) Gentile G, Greco F and Larobina D. European Polymer Journal 2013;49(12):39293936. 


\section{Acknowledgements}

The authors gratefully acknowledge the partial financial support provided by the Australian Research Council through its Centres of Excellence and Professorial Fellowships programs. 